\title{
Moving Forward
}

\section{Christopher Newland ${ }^{1}$}

Published online: 11 March 2020

(C) Association for Behavior Analysis International 2020

In 2018, the name of ABAI's flagship journal changed from The Behavior Analyst to Perspectives on Behavior Science (PoBS). Its purpose, however, remained unchanged: to inform its readership of the best science behavior analysis has to offer. I am honored to serve this mission as the second editor-in-chief of PoBS. In this role, I will continue the successful trajectory that began with the founding of The Behavior Analyst in 1978 and expanded during its transition to PoBS under the visionary stewardship of Dr. Hantula, a team of outstanding associate editors, and an increasingly diverse editorial board. This new responsibility has caused me to reflect on where the journal has been and leads me to contemplate its future.

Over the past several years, the journal has evolved - and continues to evolve - in many ways, of which I will mention two. The first is in quality and influence. PoBS articles are attracting attention both within and outside the discipline of behavior analysis. Some of this attention can be measured by the all-important, if flawed, citation impact factor. Between 2014 and 2018, PoBS's impact factor increased from 0.4 to 2.46, an astonishing rise. Although impressive, we should recognize that the impact factor is an imperfect metric of influence. It counts citations only over a short, 2-year span, and emphasizes one question: "What have you done for me lately?" Nevertheless, the impact factor is a necessary consideration for authors, especially those in the early or middle stages of their career, as they decide where to place their best work. A hard reality of modern scholarly life is that institutions of influence, including university promotion and tenure committees and extramural funding agencies, use the impact factor to evaluate the importance of an author's published work. The rise in PoBS's impact factor will, and already has, served to attract a wider range of high-quality submissions.

Other dimensions of quality are not so easily quantified. For example, perusal of PoBS reference sections reveals citations to, and influence by, work published 20, 30, or more years ago. This make sense because our science and the applications that flow from it are grounded in long-standing principles that yield an understanding of behavior as an interaction between the individual and the context in which behavior occurs, as well as a history that spans a lifetime and even generations. Such principles are difficult

M. Christopher Newland newlamc@auburn.edu 
to discover but, once isolated, they endure and should be cited. Some PoBS articles will point the way to our discipline's future, and they will do this while referencing its foundational accomplishments. There may be no quantitative measure of conceptual coherence, but this critical feature of cumulative science is important. Without it, we are merely following the latest fashion.

A second area of growth for PoBS is its ability to make contact with behavior scientists who approach topics that interest us, but do so from other directions, with different viewpoints, and novel perspectives. After all, the first word in the name of the journal is plural. No science has a monopoly on good ideas, good theories, or good methods. Some readers may be surprised at the extent to which "our" subject matter is a critical concern in other disciplines, but it is. For example, a search of the 2017 proceedings of the annual meeting of the Society for Neurosciences yields no fewer than 1,057 entries with "reward," 361 with "reinforcement," 101 with "reinforcement learning," and 12 with the exact phrase "delay discounting" (76 with "impulsivity") in their title or abstract. What insights are waiting in these papers? Can they provide a deeper understanding of our science by filling in gaps or revealing problems that we have not yet taken up? And this is but one organization. Those who engage in behavior science from sociological, developmental, linguistic, or cognitive perspectives also have much to offer us. Does it go without saying that we have much to offer them, too? Maybe not - it should be said. There are things that we do very, very well and we should trumpet these things. All of us know of examples where behavior analysts have had a major influence on social and neural sciences. PoBS can and should be a place where bridges are built and influence, in both directions, occurs.

A science of behavior has much to offer but a science of individual behavior is even more powerful. Since its inception, interest in the behavior of the individual has been baked into behavior analysis and is one of its defining characteristics. This guiding principle has yielded an impressive range of insights about how behavior works and a theoretical apparatus that massively improves our understanding of individual students, clients, animals, and many others. On reflection, it is astounding that elegant ideas that explain so much, like the matching law, the emergence of equivalence classes, delay discounting, or relapse phenomena, to name just a few examples, apply so well to and explain so much about individual behavior.

The ability to explain and reproduce the behavior of individuals yields not only profound theories but also powerful applications. Indeed, translation and application flow naturally from our ability to influence behavior, which otherwise can seem so protean, and to do so not by speculating about internal processes but by rearranging the environment in which behavior occurs. How could this ability fail to encourage investigators to translate their laboratory discoveries into application? The process of translation is rarely straightforward, however, but insights may emerge from the messiness of application. After all, basic phenomena are discovered under pristine laboratory conditions, whereas application inevitably occurs in settings in which the investigator or practitioner has far less control. This is what makes engineering such a complex and creative endeavor. Translation from the laboratory to application reveals limitations to our theories and may even highlight phenomena that our methods and theories are presently inadequate to address. This is exciting because it is the beginning of investigation and understanding and a back-and-forth that can only advance behavior science. 
We invite reviews and methodological papers on a range of topics pertinent to behavior science. We are also interested in exploring topics from different perspectives in special sections and special issues that connect a variety of papers addressing a central idea. Recent examples include a special section on substance abuse in September 2019, and a special section on cultural, behavioral, and systems science in December 2019. A special section addressing health, technology, and behavior science is planned for the June 2020 issue. Two exciting special sections are announced in this issue. One is a joint endeavor with another ABAI journal, Behavior Analysis in Practice (BAP), to publish a special section in each journal on the powerful and undervalued instructional technique, direct instruction. Papers on the science underlying direct instruction or related instructional approaches, or methodological issues in data analysis will be considered by PoBS, whereas papers on its implementation will be considered by BAP. The idea for the sections was submitted by the guest editors, William Heward and Janet Twyman, and its inspiration was derived from a symposium at the annual ABAI meeting in 2020 on the topic. The second is a special section on the application of quantitative models. The goal is to draw from the range of quantitative models that have been developed and examine how they have been applied in clinical settings as well as in other arena like behavioral pharmacology and behavior economics.

Perspectives on Behavior Science continues an arc that began over 40 years ago when The Behavior Analyst was conceived as a home for theoretical works, literature reviews, methodological papers, and topics of special interest to members of ABAI. It is a mission that PoBS editors inherit enthusiastically, and we continue will expand on so that it can serve as an outlet for basic and translational science and as two-way bridge with allied behavior sciences. The future looks exciting! 